\title{
Response of Rebar During Robotised Process of Assembling
}

\author{
Blaž Dolinšek, Researcher, (blaz.dolinsek@ikpir.fagg.uni-lj.si) \\ Prof. Dr. Janez Duhovnik (janez.duhovnik@ikpir.fagg.uni-lj.si) \\ University of Ljubljanja, Faculty of Civil and Geodetic Engineering, Institute of Structural \\ Engineering, Earthquake Engineering and Construction IT (IKPIR), \\ Jamova 2, SI - 1000 Ljubljana, Slovenia
}

\section{ABStract}

The idea of the robot system for the assembling of the reinforcement cages for reinforced concrete beams and columns and the assembling process are presented. The process of the assembling requires the moving of the rebars, which are rather flexible and therefore deformed due to their dead-weight. Additionally the accelerations during the movement could cause their oscillations. As a consequence, permanent deformations of rebars could also appear. The purpose of this paper is to present a study of the behaviour of the flexible rebars, which are exposed to the accelerations during the movement. The analyses, which enable rather accurate response of the rebars are shown. The results of the analyses are needed in the path planning of the robots, which move the rebars during the assembling process.

\section{Introduction}

Assembling of reinforcement is a construction task, which is daily carried out to a great extent. Usually, reinforcement is assembled manually at construction sites and only partially in reinforcement factories. The quality of the assembling is very changeable due to unreliability of the workers, who perform this work in the hard working conditions. One of possibilities for the achievement of better and constant quality is the usage of automation and robotisation. Although the assembling of reinforcement is a typical assembling task and it seems to be very simple, the trial of its robotisation causes several problems. They are the consequence of the defectiveness of the industrial robot's capabilities. Particularly the visual, cognitive and flexible capabilities of robots are far from the human ones. The form of the reinforcement and the process of assembling being currently performed are accommodated to the human capabilities. Therefore, the robotisation of the assembling process requires the accommodation to the robot's capabilities.
At the Institute of Structural Engineering, Earthquake Engineering and Construction IT (IKPIR) the design of the robot system for assembling of the reinforcement of the reinforced concrete beam elements has been recently carried out. The goals of the development of the robot system are to achieve as few restrictions as possible regarding the different types of the reinforcing (columns, beams, piles...), the forms of the cross sections (rectangular, T shape, I shape..), the dimensions of the reinforcement cages, and the density of the reinforcement rebars.

The process of the assembling will be described in the next chapter. It differs from the classic manual process and requires the solutions of some details which are due to the changed assembling process and which are of no importance during the classical manual process. One of these problems, discussed in the paper, is the response of the rebar during the movement in space.

\section{Short Description of the Robot System}

The robot assembling process is based on the principle of the assembling, in which the one-side-open stirrups are put on the longitudinal rebars and then the stirrups are bent in the final position [1] [2]. This solution enables the assembling of numerous reinforcement schemes. The reinforcement may consist of the bent rebars and the cross-sections of the elements may be of different shapes (rectangular, T shape, I shape...) (Figure 1). The reinforcement cage may also consist of many different stirrups in a particular cross-section.

Regarding the principle of the assembling a robot cell with robots and mechanisms needed for the performing of the assembling process was designed (Figure 2). The input elements are pre-manufactured longitudinal rebars and stirrups and the output elements are assembled reinforcement cages. 


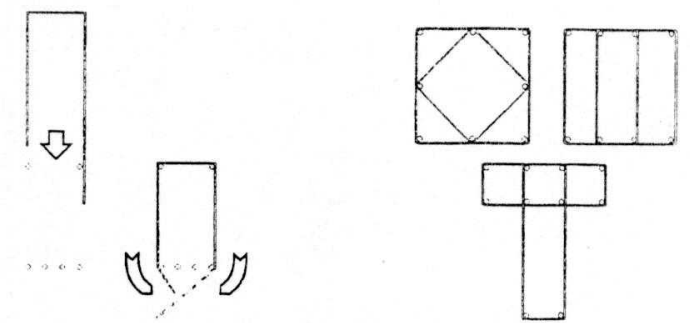

Figure 1. Principle of the assembling and various types of possible reinforcement scheme

The skeleton of the robot cell is a portal system, which is approximately $12 \mathrm{~m}$ long, $5 \mathrm{~m}$ wide and $3 \mathrm{~m}$ high space frame structure. Four robots are working in the cell: two robots for moving and assembling of rebars equipped with the grippers, a robot for bending the stirrups equipped with the bending tool, and a robot for connecting of the longitudinal rebars with the stirrups. The installation of the robots on the portal system is achieved by means of the gantry mechanisms which enable their movement in all three directions of the cell. By these means, the robots can easily reach each point inside the rectangular working area bounded by the portal system.

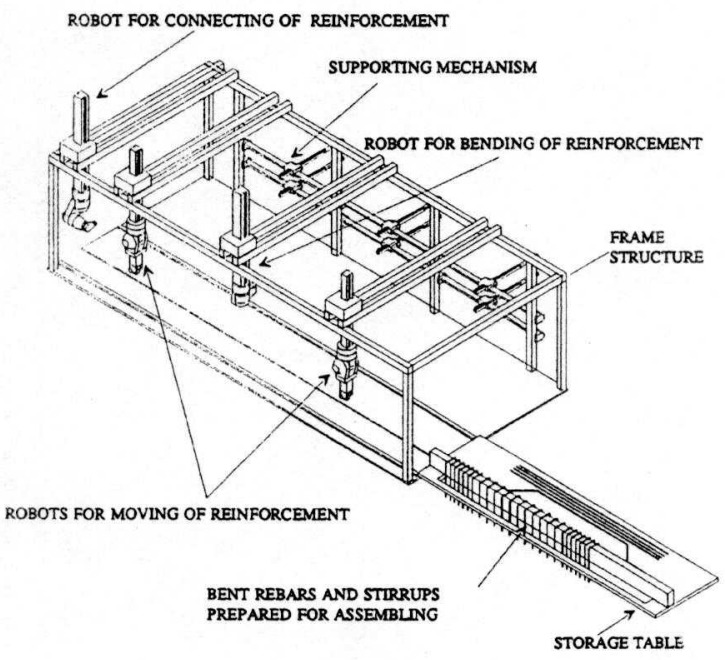

Figure 2. Robot cell

On the front side of the floor of the robot cell a storage table moves out and in the cell. Its task is to provide the prefabricated rebars and stirrups to the assembling robots and to transport the manufactured reinforcement cages out of the cell.

On the rear side of the robot cell there is a supporting mechanism (Figure 3). Its task is to support the longitudinal reinforcement in the appropriate relative distance during the assembling process. We are exploiting the fact that the longitudinal rebars in the beams and columns are placed on the particular levels. One level beam is designed for supporting one particular level of the longitudinal rebars. The level beams can move in the vertical direction. Their mutual distance defines the distance between the reinforcement levels.

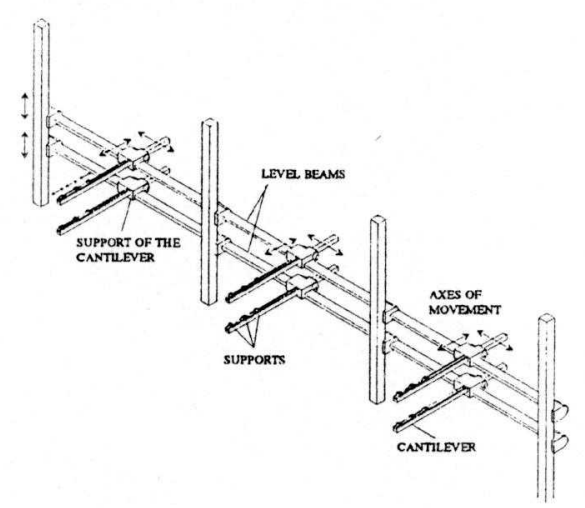

Figure 3. Supporting mechanism

The assembling process is divided in three subprocesses:

a. moving of the longitudinal rebars from the storage table to the supporting mechanism (Figure 4) and moving of the manufactured reinforcement cages from the supporting mechanism to the storage table.

b. positioning and final bending of the stirrups (Figure 5)

c. connecting of the stirrups with the rebars.

Because of the large flexibility of the longitudinal rebars and its stability during the movements, the rebar should be grasped in at least two points.

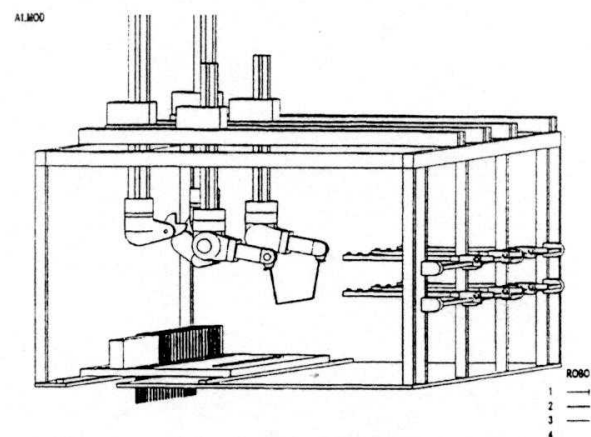

Figure 4 Moving of the longitudinal rebars to the supporting mechanism

Two robots equipped with the grippers are therefore foreseen for moving the rebar. They are equal and during the carrying of the rebars and the reinforcement cages they move symmetrically regarding the vertical cross plan of the robot cell.

The next phase of the assembling is the moving of the stirrups from the storage table to the longitudinal rebars on the supporting mechanism and its final bending. This task 
requires the collaboration of two robots: one robot for assembling and one robot for bending.

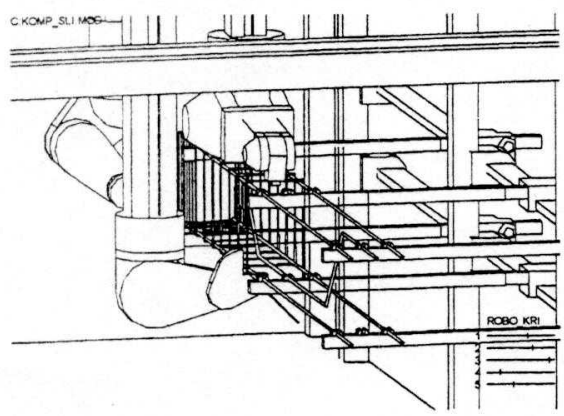

Figure 5. Final bending of stirrups

The robot for assembling grasps the stirrup, located on the storage table, and moves it near the supporting mechanism. Then it puts the stirrup from the appropriate direction on the longitudinal rebars. The robot holds it in this position, until it is not closed by the robot for bending. The robot for bending bends it with a special tool for bending. The bending is performed in many working positions which are located at the edge of the reinforcement cage and certainly also on the stirrup.

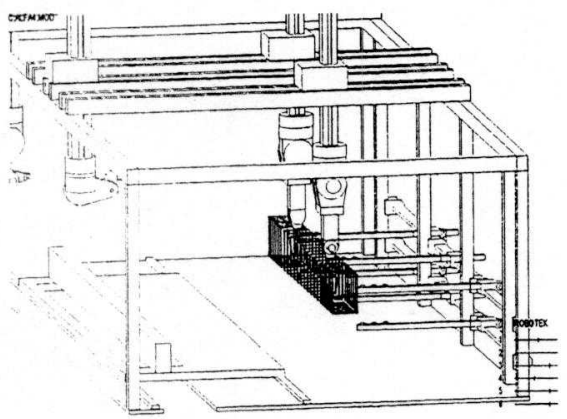

Figure 6. Moving of the reinforcement cage to the storage table

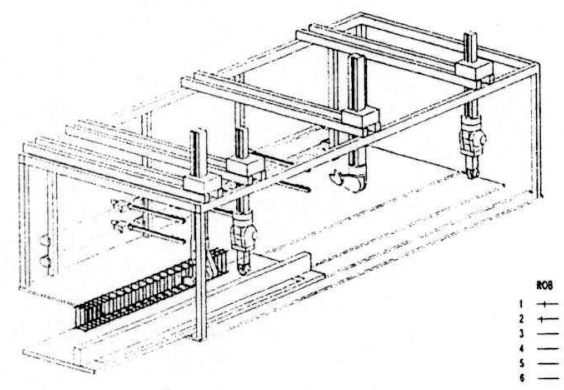

Figure 7. Carrying of assembled cage out of the robot cell

The next phase is the connecting of the stirrups with the longitudinal rebars. This task is performed by the robot equipped with the welding or the binding tool. The robot moves the tool from one working point to another around the stirrup and then to the next stirrup.

When the connecting process is finished, all robots return to the start position, while the assembling robots move the assembled reinforcement cage from the supporting mechanism to the storage table which carries the cage out from the cell to the next production stage (Figures 6 and 7).

\section{Response of the Rebars During the Movement}

The process of the assembling, described in the previous section, requires the moving of the rebars in space. The rebars differ from the objects being usually assembled by robots. Usually, the objects are rigid, but the rebars are mostly very flexible. Thus, their dead-weight could cause significant deformations at the rising from the ground. Besides, the oscillations of the rebars could appear due to the accelerations during the movement. As a consequence, permanent deformations of the rebars could also be caused. Such rebars are not suitable for the usage because of their geometrical and material imperfections. Great oscillations of the rebar are very disadvantageous. They obstruct the assembling process, and a greater space round the path of the rebar is needed to avoid the collisions with the other objects. It is very difficult to put the oscillating rebar in the accurate position. Thus, the rebar should be calmed before being laid in the final position.

Preliminary analyses of the behaviour of the rebars are therefore recommended. On the base of these analyses the path of the moving could be planned in such a way that the oscillations and plastic deformations of the rebar could not appear.

In the robotics the path planning consists of the determination of the parameters, which are needed for controlling of the robot motors in order to achieve the necessary path of the robot. Nowadays, the usage of robot simulators is the most common way for path planning. The computer programs being currently presented on the market enable the precise modelling of the particular robots, the whole robot cells, and also the design of the complete production processes. That includes also the study of the robot paths and the analysis of the loading of the robots during the movement on these paths. But usually they are not able to analyse the response of the objects during the movement. For these analyses other tools can be chosen.

In the next sections, we are presenting one of the possible ways, how to study the response of the rebar during the movement. The procedures of the analyses and the appropriate computer tools are shown. 


\subsection{Description of the Analyses of the Response}

The response of the rebar during the movement was analysed using programs for static and dynamic analyses of structures, used in structural engineering. At present, many programs are available on the market. They are mostly based on the finite element analysis. These analyses could be rather comprehensive, because the accuracy of the computing is raised by the density of the finite element mesh and by the shortening of the integration time interval. Thus, great processor power, memory capacities and computing time are required for these types of programs. In our analyses the NISA [3] program has been used. NISA consists of many modules, which enable the static and several types of dynamic analyses.

The behaviour of the various shapes of the rebars was studied. Regarding the shapes the attention was paid to the rebars, being interesting for the presented principle of the assembling. These rebars are: straight rebars, bent up rebars and opened stirrups (Figure 8). Flexible rebars, which are mostly subjected to the oscillations, are long rebars with a small diameter. Regarding the recommendations of many authors [4], the shapes of these rebars should be as simple as possible because of the alleviation of the assembling.
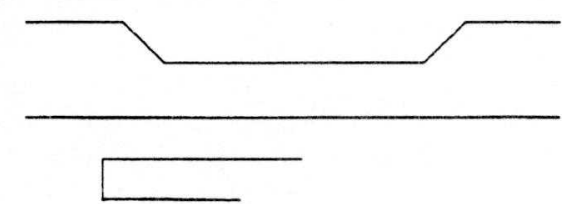

Figure 8. Shapes of the rebars, mostly used by presented principle of the assembling

The numerical results were compared with the experimental tests of the behaviours of the rebars. These tests were performed by the moving of the rebar with the real robot in the Laboratory of Robotics at the Faculty of the Electrical Engineering of the University of Ljubljana. The reduced models of the prototype rebars had to be made for the experimental tests because of the restrictions of the space in the lab. Therefore, the geometrical measure had to be reduced. Regarding the geometrical measure, all other measures of the physical quantities (time, mass ...) had to be adapted so that a full model similarity was achieved. The test of the model is shown in Figure 9.

The diameter of the tested rebar was $12 \mathrm{~mm}$, and it was $4 \mathrm{~m}$ long. It was grasped in one grasping point located at the middle of the rebar. The model of the rebar was $2 \mathrm{~m}$ long rebar with the diameter of $6 \mathrm{~mm}$. It had also additional steel rings uniformly arranged along it to ensure the model similarity with the rebar. The robot was programmed to execute the movement in the vertical direction with the quadratic course of the displacement regarding time. Thus, the velocity was linear and the acceleration was constant, about $2 \mathrm{~m} / \mathrm{s}^{2}$.

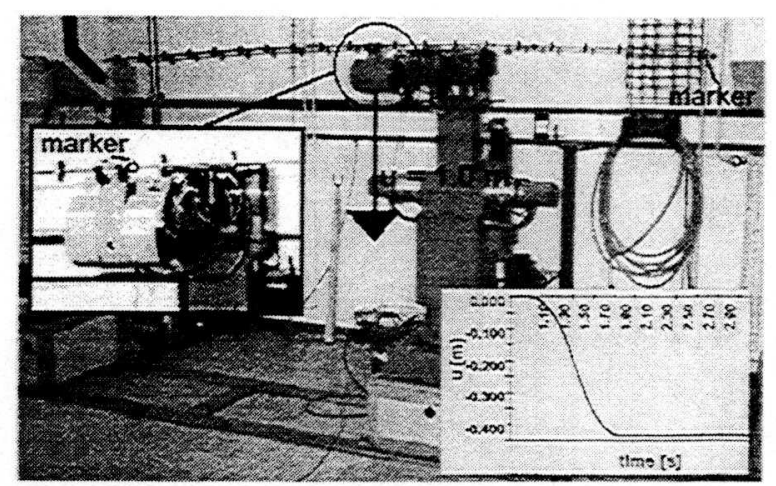

Figure 9. Experimental test of moving the rebar

The movement of the point beside the gripper and of the point at the free end of the rebar was observed. The measurements were performed by the measuring system OPTOTRAK. The markers, which transmitted infrared signals, were attached to the measuring points. The infrared signals were received by the cameras of the OPTOTRAK. The results of the measurements were the time history $3 \mathrm{D}$ position of the measuring points in the space.

In the numerical dynamic analysis of the same rebar the gripping point of the rebar was simulated as a support. The support had prescribed time history diagram of the movement as it was measured in the test. The prescribed loading was the dead-weight of the rebar.

An important information having impact on the behaviour of the rebar, is the damping of the oscillation. It was measured experimentally on the model. A graph in Figure 10 presents the damping oscillation of the free end of the rebar regarding time. The oscillations was caused by the shifting the rebar from the equilibrium position.

It could be seen that the curvature with the $0.5 \%$ damping coefficient is in the utmost agreement with the real situation. Because of the small damping, the duration of the oscillations could be of long duration. The measured damping coefficient was considered by the dynamic analyses.

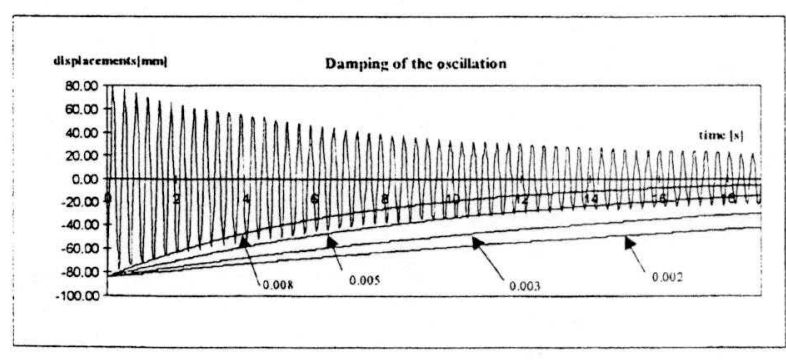

Figure 10 Damping of the oscillations 
A graph in Figure 11 presents the comparison of the experimental and analytical results of the behaviour of the rebar. The smooth curve presents the movement of the gripper and, accordingly, also the movement of the support. Two others present the movement of the point at the end of the rebar. One of them shows the experimental

\subsection{Static analysis}

Before the beginning of the movement, the grasping and the supporting points of the rebar should be determined. The grasping points are the points, in which the rebar is grasped during the movement, and the

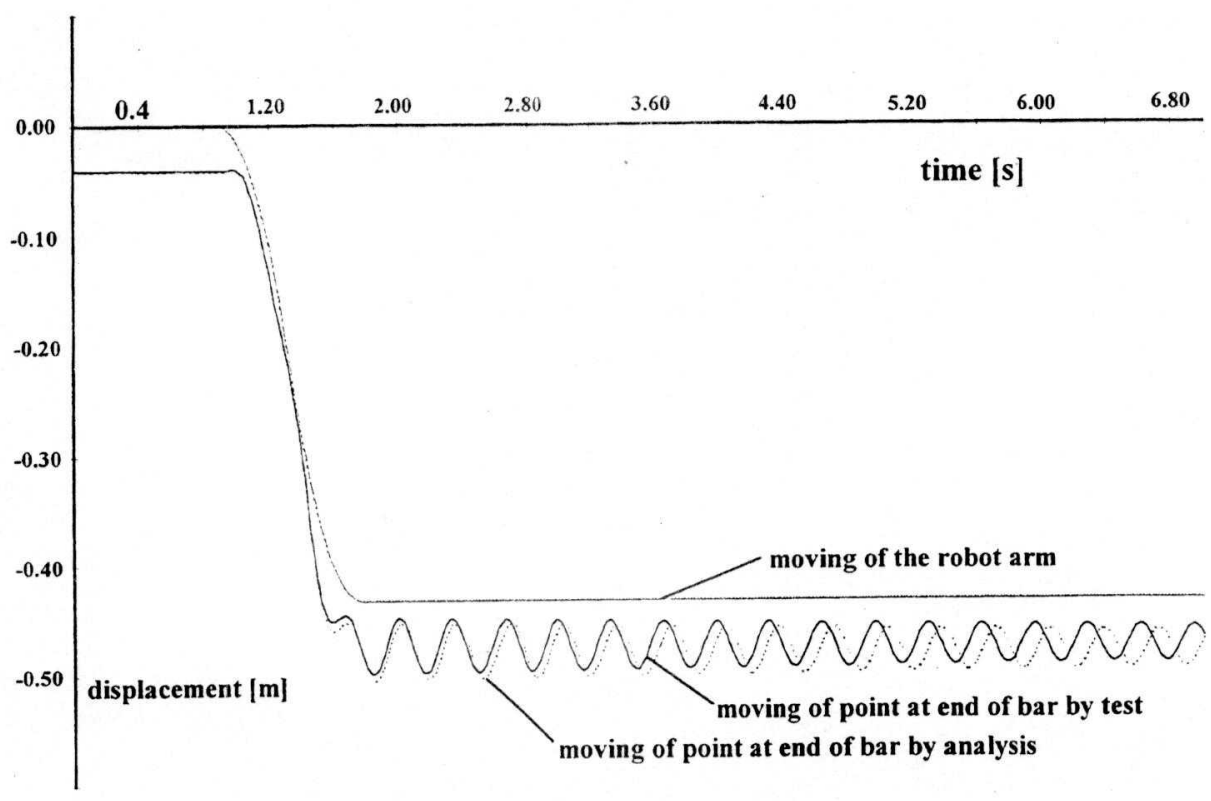

Figure 11. Comparison of the experimental and analytical results

results and the other the analytical results, obtained by the program NISA.

The accordance of the results is satisfactory. Complete accordance was impossible because of the incompleteness of the input data for the analysis. There are tolerances regarding the geometrical and material properties of the model, which could not be avoided Besides, certain tolerances are also presented in the movement of the robot.

The complete analysis of the behaviour of the rebar includes all phases of the movement: from the rising of the rebar from the ground to the laying of the rebar on the supporting mechanism. Some phases can be analysed statically, the others dynamically. The output results from the previous phase are the input data for the next phase of the computation. supporting points are the points, in which the rebar is supported on the supporting mechanism. All rebars of a particular element should have the supporting points at the same longitudinal location. The positions of the grasping points can vary from one to another rebar, but they should not coincide with the supporting points.

The supporting and the grasping points present the supports in the numerical analyses. By changing their location along the rebar the influence on the displacements of the rebar can be studied. They should be selected in such a way, that the maximum displacements along the rebar are as small as possible.

The static analysis is suitable for the determinations of the displacements, when the rebar is not moving. These situations occur at the rising of the rebar from the ground and at the laying on the supporting mechanism. The grasping and the supporting points are simulated as a full constraint supports. The loading of the rebars is their dead-weight. The analytical solutions could also be achieved for the particular shapes of the rebar. The results of the analysis are the displacements along the rebar and belonging internal forces. The stresses in the rebar should 
not exceed the yield stresses of the material. The rising of the rebar from the ground should be slowly and carefully executed, until the rebar is touching the ground. A quick activation of the dead-weight could cause significant oscillations of the rebar.

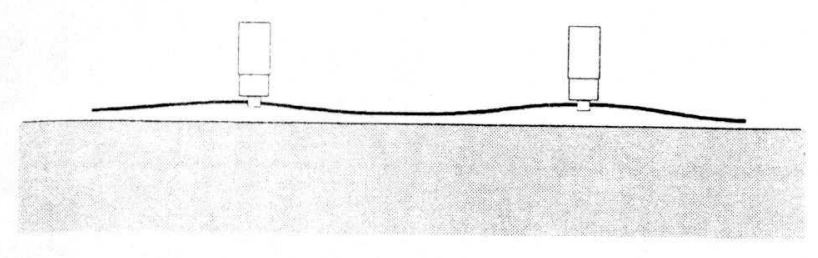

Figure 12. Rising of the rebar from the ground

\subsection{Dynamic analysis}

Dynamic analysis is needed for the study of the movement of the rebar from the storage to the supporting mechanism. The path of the movement, velocities and accelerations of the robot should be previously defined. Common robot controllers enable to select very complicated robot paths. They also enable the control of the velocities and the accelerations of the path. The ability of control depends on the types of the robot drives, on the intelligence of the controller, and on the kinematics of the robot. Ordinary robotics languages allow to select the constant accelerations on the specific path. The changing of the accelerations during the movement is the main reason for the oscillations of the rebar. The oscillations depend also on the shape and dimensions of the rebars, which influence their eigenfrequencies and mode shapes. The response of the rebar can be very different for various rebars. Oscillations of the rebar cause also the changeable actions to the robot arm. The dynamic analysis enables the studying all of these influences. The transient dynamic analysis can be used. It can be linear or non-linear. The non-linear one enables also the studying of the plastification of the material. However, in our case the plastic deformations of the rebar are not allowed, therefore the linear analysis was used, but the yield strength of the material should not be exceeded. Using time integration methods, the whole time is divided into small intervals. The analysis advances step by step. In each time interval the displacements, the velocities, the accelerations and the internal forces are calculated for each point of the rebar. The accuracy and the stability of the analysis depend on the time interval and on the accuracy of the input data.

The damping matrix should be given as a linear combination of the mass and stiffness matrix. It could be determined by means of the eigenfrequencies of the rebar. The eigenfrequencies depend on the geometry of the rebar, on the mass and on the grasping points, which present the supports. The supports are simulated as a full constraint supports. Thus, the parts of the rebars on each side of the support can oscillate independently and they can have a different damping matrix.

The dynamic analysis enables the searching of the most appropriate combinations of the grasping points, the path, the velocities and the accelerations of the movement. The ability of the robots to achieve these path parameters can also be tested.

The scheme in Figure 13 presents the flow chart for the determination of the dynamic response of the rebar.

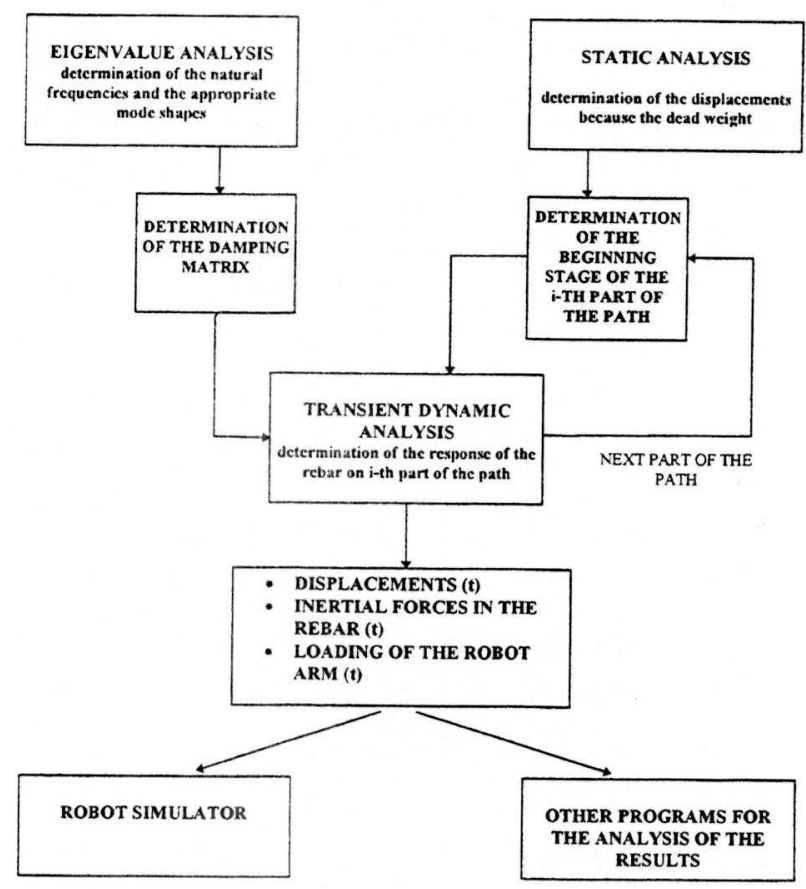

Figure 13. Flow chart of the analyses

The beginning stage is the static equilibrium stage at the time of the rising of the rebar from the ground. These stages can be determined using static analysis. By means of the eigenvalue analysis, the damping matrix can be determined. If the path is long and complicated, it can be divided into many sub-paths. The beginning stage of each sub-path is the ending stage of the previous sub-path.

The results of the dynamic analysis are the time history of displacements, the internal forces of the rebars, and the action forces of the robot grippers.

\section{Examples of the Analyses}

As the first example, the results of the analysis of the response of the bent up rebar are presented. The diameter of the rebar was $8 \mathrm{~mm}$ and it was $5 \mathrm{~m}$ long. The moving path was two dimensional (Figure 14), $1 \mathrm{~m}$ in vertical direction and then $1 \mathrm{~m}$ in horizontal direction. The accelerations and the decelerations of the movement was constant $2 \mathrm{~m} / \mathrm{s}^{2}$. 


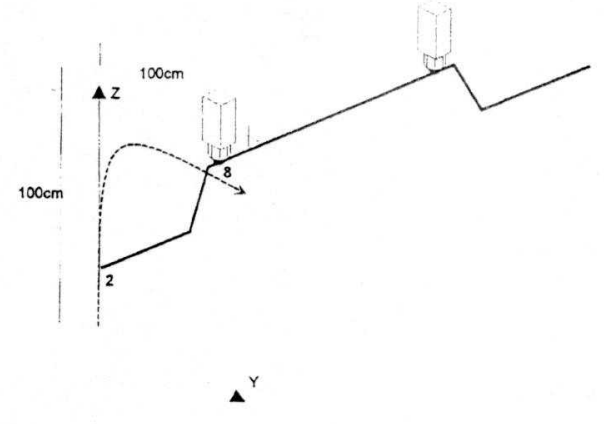

Figure 14. Path of the moving

The graph in Figure 15 presents the time history of the displacements for two characteristic points of the rebar: a point beside the gripper and a point at the free end of the rebar (Figure 14). The rebar begins to move after one second equally accelerated in the vertical ( $Z$ ) direction. When it achieves half length of the vertical path, it begins to move with equal deceleration in $\mathrm{Z}$ direction, and, at the same time, it begins to move in the horizontal direction in the same way as in the vertical direction. The point 2 was at the beginning in the static equilibrium stage (P2_UZ and P2_Uy). After the execution of the moving the point oscillates in both directions and it slowly $(0.5 \%$ damping) approaches its static equilibrium stage.

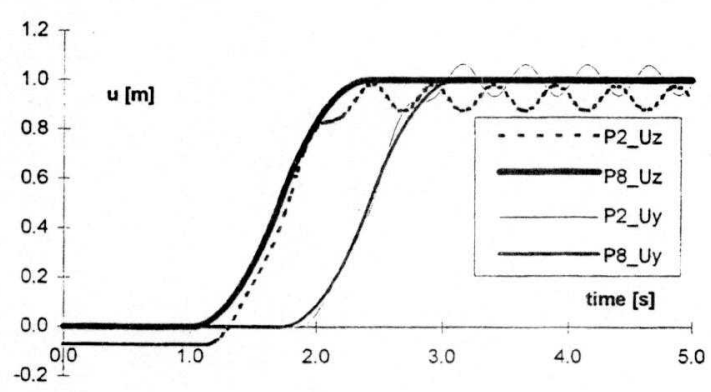

Figure 15. Time history of the displacements

Next diagrams (Figures 16) shows the time history of the velocity and acceleration for the same points of the rebar.

Only diagrams for quantities in vertical directions are shown. The shape of the adequate diagrams in horizontal directions are similar, but they are shifted for the adequate time interval in the right.

The diagrams in Figure 17 present the envelope of the maximal moments along the rebar during the movement. The maximal moments should not exceed the plastic moment. The resulting moment at a specific location on the rebar is the vector sum of the $\mathrm{My}$ and $\mathrm{Mz}$ components.
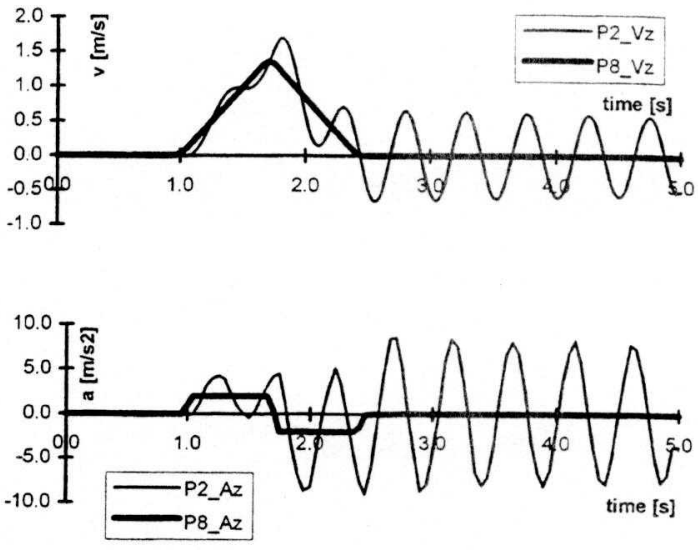

Figure 16. Time history of the velocities and the accelerations
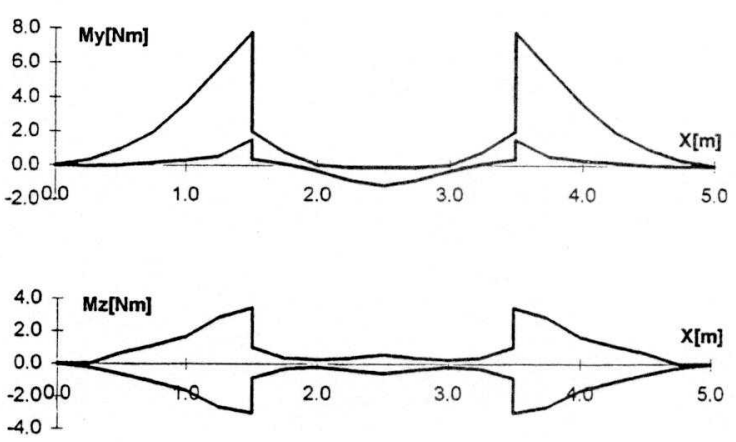

Figure 17. Moment envelope along the rebar during the movement

For the selected positions of the grasping points the maximum moments appear beside the grasping points (point 8). The moment history in these points is shown in Figure 18. At the beginning the moments are the same as the static moments due to the dead weight.

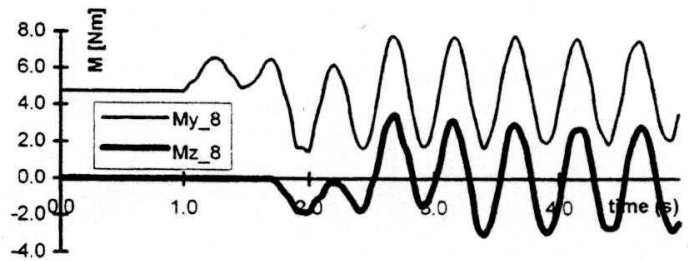

Figure 18. Time history of moments in the rebar near the grasping points

The differences of the moments and the shear forces between the points at the left and the right side of the grasping points present the supporting reactions. 
Supporting reactions in our case present the loading of the robot arm. At the beginning the forces and the moments are the same as the static reactions, but later on they are in disorder. The reason is in the independent oscillations of the parts of the rebar at the each side of the grasping points.
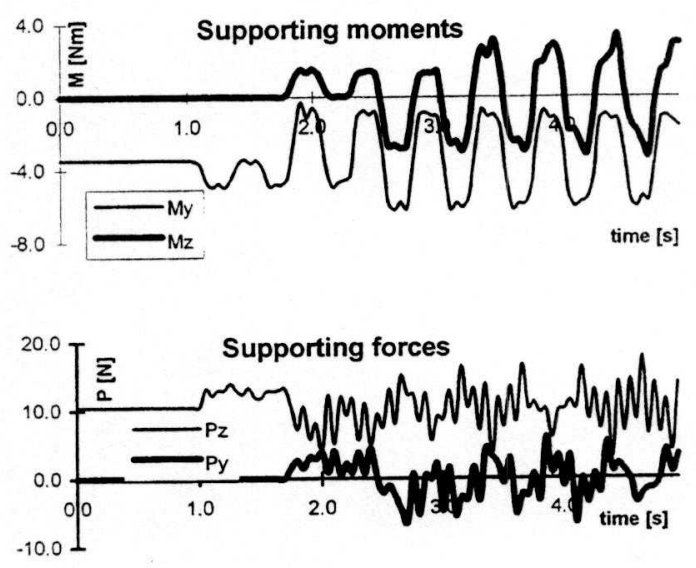

Figure 19. Time history of the robot loading

The movement of the presented rebar causes the oscillations of the rebar, which last a lot of time. If the in the previous example. The diameter of the rebar and the accelerations were changed. The grasping points was chosen in such a way that the displacements because of its dead-weight at the end of the rebar and the deformation in the middle of the rebar were equal.

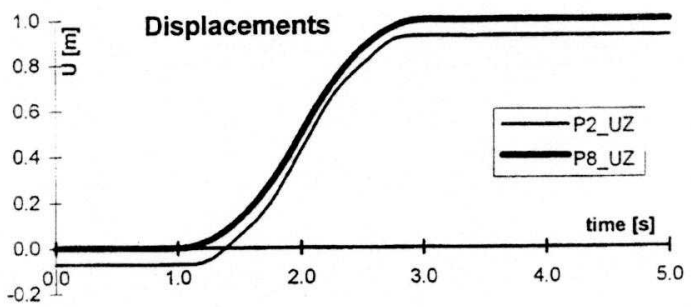

Figure 20. Response of the rebar in the movement with the small accelerations

The diagram in Figure 21 shows the displacements of the free ends of the rebar, if the diameter was changed and the acceleration was $5 \mathrm{~m} / \mathrm{s}^{2}$. The amplitudes of the rebars with the diameters 16 and $20 \mathrm{~mm}$ are quite negligible.

The maximum moments in the rebars increase with the increasing of the diameter, due to the increased mass of the rebar and the load capacity of the rebars increases as

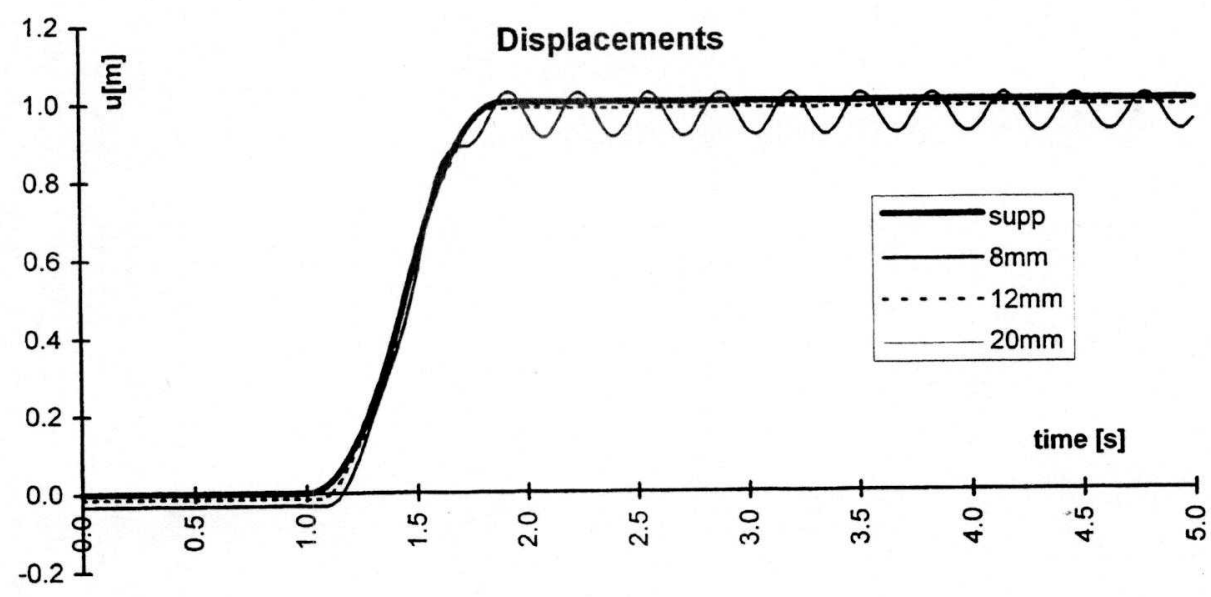

Figure 21. Influence of the diameter on the displacement history.

same rebar moves with the smallest acceleration on the same path, the oscillations in the rebar can be almost negligible. The graph in Figure 20 shows such an example. The acceleration was $1 \mathrm{~m} / \mathrm{s}^{2}$. The displacement of the point 2 is nearly the same during the whole time of the movement.

The diagrams in Figures 21 and 22 show the results of a parametric study of a straight rebar. It was $6 \mathrm{~m}$ long and the characteristics of the movement were the same as well (Figure 22, upper graph). A suitable measure criterion could be the relationship between the moment and the yield moment (Figure 22, lover graph). The picture is now inverse regarding the upper graph. The slender rebars are much closer to its yield border than the thicker ones.

The Figure 23 shows the response of the rebar in the movement along the same path, but with various accelerations. The diameter of the rebar was $12 \mathrm{~mm}$. The time history of the vertical displacements of the free edge 
of the rebar is presented. It is shown that the greater accelerations do not always cause the greater oscillations
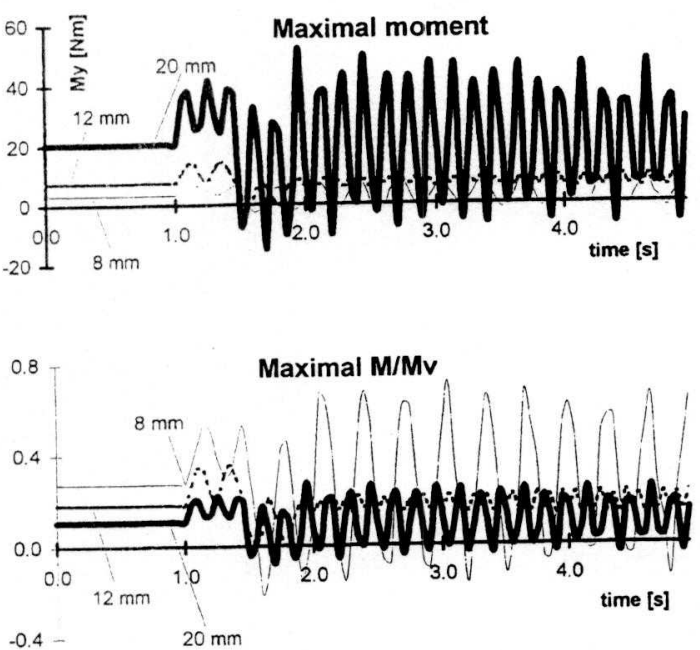

Figure 22. Influence of the diameter on the moment history.

of the rebar. Oscillations depends more on the resonance of the rebar. The response at the acceleration $10 \mathrm{~m} / \mathrm{s}^{2}$ is nearly negligible.

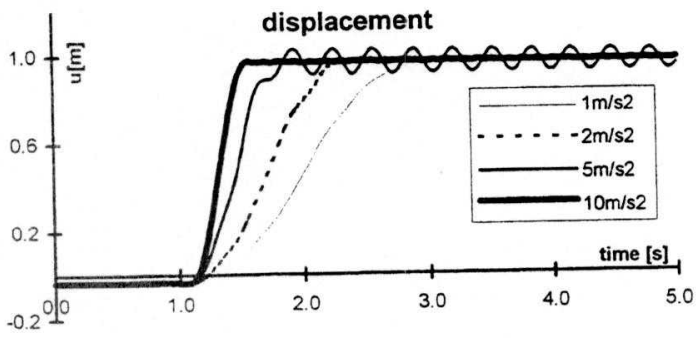

Figure 23. Influence of the acceleration on the displacement history.

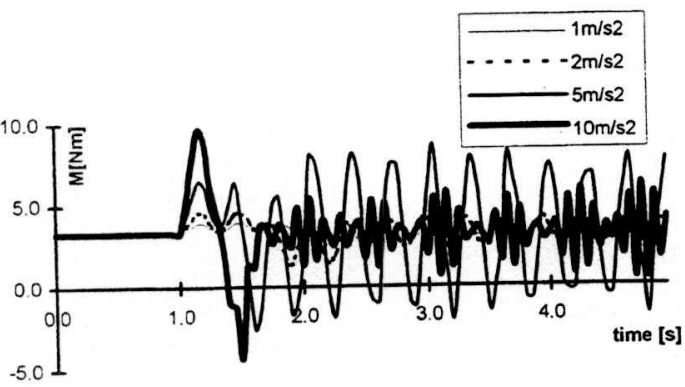

Figure 24. Influence of the accelerations on the moment history

Figure 24 shows the time history of the maximum moment. It is proportional to the displacement history. The acceleration $5 \mathrm{~m} / \mathrm{s}^{2}$ causes the greatest oscillations of the moments in the rebar. The greatest moments appear in the movement with the acceleration $10 \mathrm{~m} / \mathrm{s}^{2}$, but at the end of the moving they reduce very quickly.

\section{Use of the Results of the Analyses}

The results of the analyses can be used for the optimisation of the paths of the movement. These paths with their parameters (shape of the path, velocities and accelerations) should enable the fastest way and the satisfying accuracy of the assembling. The rebars should also remain undamaged. The moving should not be too fast due to the appearing of the oscillations of the rebar. But the assembling velocity should not be too slow as well, because of the decreasing of the efficacy of the robot system.

To avoid of the oscillations of the rebars during the movement is the most important goal of the path planning. This can be achieved by the small accelerations on the path or by the continuous course of the accelerations. The latter way demands the capable robots and their controllers. If the oscillations of the rebar appear, they should be damped before the laying of the rebar in the final position. The most simple way is waiting but it takes a long time because of very low damping of the steel. Other solutions are possible as well. One of the advanced solutions could be the smart controlling of the robot arm. The robot arm could cause short pushes in the direction of the actions caused by the rebar to the robot. The tender pressure on the oscillating part of the rebar can also damp the oscillations.

The accurate course of the oscillation is not essential for the assembling. It is almost impossible to determine it because of the inaccurate input data. The useful results are the amplitudes and the duration of the oscillations. By means of the amplitudes the space around the ideal position of the rebar can be determined, where the rebar can appear during the movement. To avoid the collisions no objects should be in this area. The duration of the acceleration enables to determine the waiting periods during the particular phases of the assembling. The results for the loading of the robot arm during the movement are also very significant. They enable more precise determination of the loading of the robot drives.

The searching of the most suitable path is not explicit. Many different variants should be tested. The changeable parameters are the positions of the grasping points, shape of the path, and the accelerations on the paths.

The position and the quantity of the reinforcement in the reinforced concrete elements is changing steadily. In spite of this fact the particular rebars are repeated because of the restriction of the shapes and of the modularity of the structures. The paths of the moving from the storage table to the supporting mechanism are also repeated. Therefore, 
the storage of the analysed examples in a database is reasonable. The database will be steadily supplemented by the new examples. Besides, a capable expert system will also be needed. It should be capable to use and complete the database, to use the programs for static and dynamic analyses, to value the results of the analyses and to predict the results on the base of the examples analysed. In this way the comprehensive analyses can be avoided and the automation of the path planning can be achieved.

The path should be analysed and defined in the preliminary stage. It should be included into the process planning, which is performed by the robot simulator. Thus, the robot simulator and expert system can collaborate with and supplement each other. For the particular path the robot simulator can analyse the response of the robots and their control parameters, and the expert system can analyse the response of the rebar. In the preliminary graphical simulations of the process, the expert system can supplement the simulation with the data of the real behaviour of the rebar and the robot simulator can test the possible collisions of the rebar with the remaining objects in the robot cell.

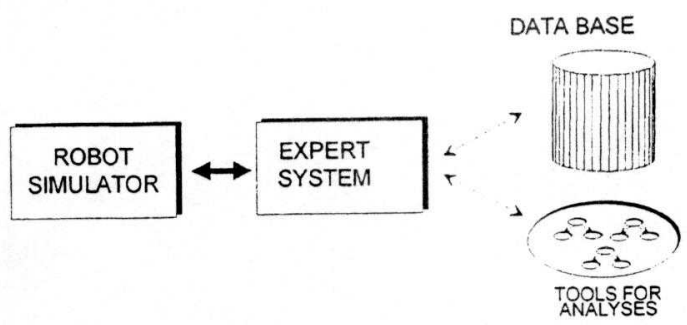

Figure 25. Expert system for the path planning

\section{Conclusion}

The experimental and numerical results show that the preliminary analyses of the behaviour of the rebar are needed in the planning of the assembling process. The results can be used in the path planning process of the robots. Thereby, the harm of the rebars, and the collisions can be avoided. They enable also the determination of the most optimal path of the moving.

The programs for structural analysis can be used for the research of the behaviour of the rebars during the movement. The accuracy of the analyses requires great capabilities of computers. Certain inaccuracy of the input data can not be avoided, therefore, the exaggeration regarding the accuracy is also senseless. Due to the repeating of the shapes and dimensions of the rebar, the building of the database of the analysed examples will be reasonable. With an appropriate expert system, the automation of the path planning could also be achieved

The robot system is still in its developing stage. At present, it is studied by the robot simulator
WORKSPACE, where the optimisation regarding the construction and kinematics of the particular robot is done. The paper is limited to discuss only one of the problems, which is caused by the new assembling process. We are trying to solve some of the problems which could be found out by the simulation.

More problems will appear in the building of the real prototype. Beside the others, the financial problems is the most important. The present recession in the construction industry in some states is not favourable to invest into the research and development. The current capacities of the reinforcement factories are not enough exploited at present. Therefore, we are interested in the cooperation with the manufacturers, who have experience with the robotisation in construction and who consider that the presented idea of the robotised assembling is interesting to realise.

\section{Acknowledgement}

This work was financially supported by The Ministry of Science and Technology of the Republic of Slovenia and by the EU as a part of TEMPUS JEP 3008 ICADERS. Both supports are gratefully acknowledged.

\section{References}

[1] J. Duhovnik, B. Dolinšek, Computer Modelling of Robotic Assembling of Reinforcement, Construction on the Information Highway, CIB Workshop W78 "Working Comission on Information Technology in Construction" and TG 10 "Task Group on Computer representation of Design Standards and Building Codes", Bled, 10.-

12.6.1996, Proceedings, edited by $\breve{Z}$. Turk, str. 145-152 (1996)

[2] B. Dolinšek, Master Thesis, Faculty of Civil and Geodetic Engineering, University of Ljubljana, (1996).

[3] NISA, Users Manual, Michigan, USA (1993).

[4] CEB, Industrialisation of Reinforcement in Reinforced Concrete Structures, Synthesis report prepared by Task Group VII/3of the CEB, Lausanne, Switzerland, (1985) 